\title{
Hippocampal microglial activation triggers a neurotoxic-specific astrocyte response and mediates etomidate-induced long-term synaptic inhibition
}

\author{
Dongliang $\mathrm{Li}^{1 \dagger}$, Mingming Chen ${ }^{2 \dagger}$, Tao Meng ${ }^{1}$ and Jianchun Fei ${ }^{{ }^{*}}$
}

\begin{abstract}
Background: Accumulating evidence has highlighted the importance of microglial and astrocyte responses in the pathological development of postoperative cognitive dysfunction (POCD). However, the mechanisms involved are not well understood.

Methods: A perioperative neurocognitive disorders (PND) mouse model was generated by administering etomidate, and cognitive function was assessed using the Morris water maze and novel object recognition tests. Excitatory and inhibitory postsynaptic currents were recorded to analyze neuronal activity. In addition, microglia and astrocytes were isolated by magnetic-activated cell sorting, and genes that were activated in these cells were identified using quantitative polymerase chain reaction.

Results: We observed dramatic cognitive impairment at 1 and 3 weeks after etomidate was administered to 18 month-old mice. Microglia and astrocytes isolated from the hippocampus showed significant microglial activation during the early pathological stage (i.e., 1 week after etomidate injection) and an A1-specific astrocyte response during the late pathological stage (i.e., 3 weeks after etomidate injection). Furthermore, when microglia were eliminated before etomidate was injected, the A1-specific astrocyte activation response was significantly reduced, and cognitive function improved. However, when microglia were eliminated after etomidate application, astrocyte activation and cognitive function were not significantly altered. In addition, activating microglia immediately after a sedative dose of etomidate was injected markedly increased A1-specific astrocyte activation and cognitive dysfunction.
\end{abstract}

Conclusions: A1-specific astrocyte activation is triggered by activated microglia during the initial pathological stage of PND and induces long-term synaptic inhibition and cognitive deficiencies. These results improve our understanding of how PND develops and may suggest therapeutic targets.

Keywords: General anesthetics, Inflammation, Synaptic plasticity, IPSC, EPSC, magnetic-activated cell-sorting

\footnotetext{
* Correspondence: qlfeijc@163.com

${ }^{\dagger}$ Dongliang Li and Mingming Chen contributed equally to this work.

'Department of Anesthesiology, Qilu Hospital of Shandong University, Ji'nan 250012, China

Full list of author information is available at the end of the article
}

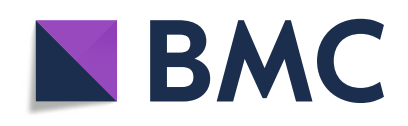

( ) The Author(s). 2020 Open Access This article is licensed under a Creative Commons Attribution 4.0 International License, which permits use, sharing, adaptation, distribution and reproduction in any medium or format, as long as you give appropriate credit to the original author(s) and the source, provide a link to the Creative Commons licence, and indicate if changes were made. The images or other third party material in this article are included in the article's Creative Commons licence, unless indicated otherwise in a credit line to the material. If material is not included in the article's Creative Commons licence and your intended use is not permitted by statutory regulation or exceeds the permitted use, you will need to obtain permission directly from the copyright holder. To view a copy of this licence, visit http://creativecommons.org/licenses/by/4.0/. The Creative Commons Public Domain Dedication waiver (http://creativecommons.org/publicdomain/zero/1.0/) applies to the data made available in this article, unless otherwise stated in a credit line to the data. 


\section{Background}

General anesthetics may induce postoperative cognitive dysfunction (POCD), now progressively recognized as perioperative neurocognitive disorders (PND), which occurs mainly among elderly patients and is characterized by cognitive deficiencies, memory loss, and decreased quality of life [1-5]. Despite excellent research in fiber photometrics, optogenetics, and chemogenetics showing how clinical anesthetics affect the central nervous system and produce side effects [6], the precise mechanisms involved remain elusive.

Current data suggest that all the effects of general anesthetics are derived from synaptic inhibition [7, 8], including forms of inhibition that are essential (e.g., loss of consciousness and immobility during surgery) and forms of inhibition that are undesired (e.g., subsequent cognitive impairment and memory loss). Synaptic inhibition produces transient inhibitory currents and occurs due to dysfunction of ligand-gated ion channels and neurotransmission. This, in turn, is due to a high concentration of $\gamma$-aminobutyric acid (GABA) in the synaptic cleft, which activates postsynaptic terminal GABA type A receptor (GABAA-R) channels [9, 10]. In addition, persistent synaptic inhibition, which results in an inhibitory current after the anesthetic has been eliminated, is probably due to complex effects produced by cytokines that are secreted by astrocytes, which express GABAARs on the synaptic surface [10-14]. However, little is known about the initial events that trigger the neurotoxic astrocytic response.

Accumulating evidence has suggested an important role for microglial activation in triggering the neurotoxic astrocytic response (i.e., A1-specific reactivation). For example, activated microglia induced A1-specific astrocytic responses by secreting multiple cytokines (including interleukin [IL]-1 $\alpha$, tumor necrosis factor [TNF] $\alpha$, and C1q), whereas lipopolysaccharide (LPS) failed to induce such responses in Csf1r knockout mice lacking microglia and impair synaptic plasticity [15]. Furthermore, A1-specific astrocytes activated by microglia have also been detected in neurodegenerative transgenic mice (e.g., 5XFAD, R6/2, and SOD1-G93A mice) and shown to propagate inflammatory effects [16]. In addition, suppressing the activation of microglia and neuroinflammation have been identified as promising approaches for improving cognitive function after general anesthesia [17-19]. Therefore, we suspect that microglial activation is involved in longterm cognitive dysfunction, mainly by triggering neurotoxic astrocytic responses.

To test our hypothesis, we established PND mice using etomidate injections and investigated microglial and astrocyte responses. We observed significant nonspecific microglial activation at 1 week and A1-specific astrocyte activation at 3 weeks after etomidate injection. Next, we eliminated the microglia using pexidartinib (PLX3397) and further assessed the astrocytes and cognitive function. The data showed that microglial activation had to be suppressed during the initial pathological stage to prevent A1-specific astrocyte induction and rescue cognitive dysfunction. Finally, we found that activating microglia before a sedative dose of etomidate was administered was sufficient to trigger A1-specific astrocytic responses and impair cognitive function. These data suggest that microglial activation during the early pathological stage induces long-term cognitive dysfunction by triggering A1-specific astrocytic changes and sustained synaptic inhibition.

\section{Methods \\ Animals}

In this study, male Thy1-egfp mice purchased from Jackson Labratories (Bar Harbor, ME, USA) were used. All animals were fed in a standard mouse chow and water ad libitum, housed in the animal center of Shandong University with a temperature and humidity-controlled environment, and under a 12:12 light: dark cycle. All procedures were approved by the Administration Committee of Experimental Animals, Shandong Province and Shandong University.

\section{Anesthesia}

According to the data from previous study [20] in establishing the relationship between loss of righting reflex and effective dose $\left(\mathrm{ED}_{50}\right.$ and $\left.\mathrm{ED}_{100}\right)$, the anesthetizing and sedative dosage of etomidate were set as $20 \mathrm{mg} / \mathrm{kg}$, i.p. and $8 \mathrm{mg} / \mathrm{kg}$, i.p. respectively in aged (18-month-old) mice. At the same time, aged (18-month-old) mice received equal volume of physiological saline (i.p.) were designated for control. To avoid hypothermia or hypoxia, we monitored the body temperature and heart rate by pulse oximetry sensor (MouseOx, Starr Life Sciences Corp., USA). After general anesthetics, mice were allowed to recovery in a temperature controlled (35 \pm 1 $\left.{ }^{\circ} \mathrm{C}\right)$ acrylic chamber flushed with medical air $\left(30 \% \mathrm{O}_{2}\right.$, $70 \%$ air).

\section{Behavioral function test}

Behavioral function test (12 mice per group) including Morris water maze (MWM) test and novel object recognition (NOR) test were performed according to previous study [21].

In detail, MWM test is divided into three sessions and lasts 8 days ( 4 trails per day). Before the test, a black plastic pool (100 cm in diameter and $40 \mathrm{~cm}$ in height) was filled with water (adding white food color and controlling temperature at $20 \pm 1{ }^{\circ} \mathrm{C}$ ). At day 1 and day 2 (visible platform training), we placed platform $0.5 \mathrm{~cm}$ 
above the water surface and placed mice in the opposite quadrant to platform quadrant. At day 3 to day 7 (hidden platform training), we transfer platform to the opposite quadrant compared to visible platform test and filled water to let water surface $0.5 \mathrm{~cm}$ over platform, then mice were placed in the opposite quadrant to platform quadrant. From day 1 to day 7, mice were allowed to swim and find platform for escaping from water in 60 seconds per trail. If mice fail to find platform in 60 seconds, we lead them swimming to the platform by a stick and allow them stay in the platform for 10 seconds. At day 8 (transfer test), we remove platform from pool and placed mice in the opposite quadrant to platform quadrant and allow mice swimming in the pool for 90 seconds. During each trail, swim path of mice were recorded and average swimming speed $(\mathrm{m} / \mathrm{sec})$ and time $(\mathrm{s})$ required to reach the platform were calculated by ANY-maze (Global Biotech Inc., NJ, USA).

Novel object recognition test is divided into 3 sessions in an open field $(50 \mathrm{~cm}$ in length, $50 \mathrm{~cm}$ in width, $40 \mathrm{~cm}$ in height) and lasts for 3 days. At day 1 (habituation session), mice were placed in the center of open field and allowed to adapt the context for 10 min. At day 2 (acquisition session), mice were placed in the center of open field with two identical objects in it and allowed to recognize objects for $5 \mathrm{~min}$. At day 3 (testing phase), we first replace one of the object with a novel object different in shape and color and then placed mice in the center of open field and allowed them to recognize objects for $5 \mathrm{~min}$. We recorded the whole test by ANY-MAZE software (Global Biotech Inc., NJ, USA) and further analyzed total exploration time (s) and the discrimination index (DI, $\mathrm{DI}=$ (time exploring the novel object- time exploring the familiar object)/total exploration time).

\section{Electrophysiological analysis}

The patch clamp whole-cell recording were applied to pyramidal neurons in DG region and EPSC and IPSC were detected and analyzed according to the protocol from previous study [22]. In brief, 350 um-thick brain slices were prepared in pre-cold oxygenated $\left(95 \% \mathrm{O}_{2} /\right.$ $5 \% \mathrm{CO}_{2}$ for more than 2 hours) artificial cerebrospinal fluid (high-sucrose version of aCSF: $87 \mathrm{mM} \mathrm{NaCl}$, $2.5 \mathrm{mM} \mathrm{KCl}, 7 \mathrm{mM} \mathrm{MgCl}, 1.25 \mathrm{mM} \mathrm{NaH}_{2} \mathrm{PO}_{4}, 25 \mathrm{mM}$ $\mathrm{NaHCO}_{3}$, and $25 \mathrm{mM}$ glucose, $75 \mathrm{mM}$ sucrose, $\mathrm{pH} 7.3$ ). Then, transferred brain slices into a culture chamber and recovered for 2 hours, perfusing the chamber with oxygenated aCSF $(120 \mathrm{mM} \mathrm{NaCl}, 3 \mathrm{mM} \mathrm{KCl}, 4 \mathrm{mM}$ $\mathrm{MgCl}_{2}, 1 \mathrm{mM} \mathrm{NaH} \mathrm{PO}_{4}, 26 \mathrm{mM} \mathrm{NaHCO}$, and $10 \mathrm{mM}$ glucose, $\mathrm{pH}$ 7.3) and maintained temperature between $32-34{ }^{\circ} \mathrm{C}$. After that, we finally transferred brain slice into recording chamber (perfused with aCSF and maintained temperature between $32-34{ }^{\circ} \mathrm{C}$ ) and pyramidal neurons in DG region and EPSC and IPSC were detected by micropipette were filled with working buffer ( $1 \mathrm{mM} \mathrm{MgCl} 2,0.2 \mathrm{mM}$ EGTA, $4 \mathrm{mM}$ Mg-ATP, $0.3 \mathrm{mM}$ $\mathrm{Na}-\mathrm{GTP}, 125 \mathrm{mM}$ Cs- methanesulfonate, $5 \mathrm{mM} \mathrm{CsCl}$, $10 \mathrm{mM}$ phosphocreatine and 5mM QX314; $\mathrm{pH} 7.3,285$ mOsm), under an upright microscope equipped with a $40 \times$ water-immersion lens (Axioskop 2 Plus, Zeiss). The sEPSC $(-60 \mathrm{mV})$ and sIPSC $(0 \mathrm{mV})$ data were obtained by MultiClamp 700B amplifier and 1440A digitizer (Molecular Device, USA). Then, mEPSC ( $-60 \mathrm{mV})$ and mIPSC $(0 \mathrm{mV})$ data were obtained in the presence of 1 $\mu$ um TTX (MCE, China).

\section{Magnetic-activated cell-sorting (MACS)}

To assess the response state of microglia and astrocyte in hippocampus, we isolated astrocyte by using magnetic-activated cell-sorting. First, hippocampus were separated from mice and immediately incubated with digestion buffer $(300 \mu \mathrm{g} / \mathrm{ml}$ DNasel (Sigma-Aldrich, USA) and $1 \mathrm{mg} / \mathrm{ml}$ papain (MCE, China) at $37{ }^{\circ} \mathrm{C}$ for $30 \mathrm{mi}-$ nutes. After that, we centrifuged lyses at $300 \mathrm{~g}$ for $15 \mathrm{mi}$ nutes and collected cell pellets for microglia and astrocyte isolation according to the manufacturer's instructions of CD11b (Microglia) MicroBeads and GlastMicroBeads kit (Miltenyi Biotech, Germany) respectively.

\section{Flow cytometry}

Flow cytometry was used to confirm the purity of microglia and astrocyte after MACS and the number of microglia in hippocampus after PLX3397 chow. The hippocampus separated from mice were immediately incubated with digestion buffer $(300 \mathrm{ug} / \mathrm{ml} \mathrm{DNa-}$ sel (Sigma-Aldrich, USA) and $1 \mathrm{mg} / \mathrm{ml}$ papain (MCE, China) at $37{ }^{\circ} \mathrm{C}$ for 30 minutes. After that, we centrifuged lyses at $300 \mathrm{~g}$ for 15 minutes and collected cell pellets. The cell pellets together with ones obtained after MACS then were suspended with phosphate buffer saline (PBS) buffer and incubated with corresponding antibody (anti-CD11b-pe (Miltenyi Biotech, Germany) and anti-CD45-APC (Miltenyi Biotech, Germany) for microglia; anti-GLAST-APC (Miltenyi Biotech, Germany) for astrocyte) and prepared for flow cytometry assay by Attune NxT system (BD Biosciences, USA).

\section{Quantitative reverse transcription (RT) quantitative PCR}

As previously described [23], total RNA from hippocampus was extracted by using TRIzol reagent (Invitrogen, Carlsbad, CA, USA) and reverse-transcribed under standard conditions using the PrimeScript ${ }^{\mathrm{TM}} \mathrm{RT}$ Reagent Kit (Takara, Tokyo, Japan). After that, the gene level was detected using the StepOnePlus ${ }^{\mathrm{TM}}$ System 
(Applied Biosystems, Carlsbad, CA, USA) with SYBR Premix Ex Taq ${ }^{\text {Tu }}$ II (Takara) and the appropriate primers (Table S1) and Gapdh was used as the internal control.

\section{Adeno-associated virus (AAV) stereotaxic injection and CNO treatment}

Designer receptors exclusively activated by designer drugs (DREADDs) application were performed as previous study described [24]. Chemogenetical virus AAV2/ 9-Gfap-hM4D(Gi)-mCherry-WPRE-pA (titer, $1.72 \times$ $1013 \mathrm{vg} / \mathrm{mL}$ ) was purchased from HANBIO Technology (Shanghai, China) and injected into the hippocampus of mice under anesthetic state which is induced by isoflurane $(1.3 \%)$ for as much time as it takes and fixed on a stereotaxic apparatus (RWD, Shenzhen, China). The injected coordinate are AP, $-2.00 \mathrm{~mm}$; ML, $\pm 1.6 \mathrm{~mm}$; $\mathrm{DV},-2.0 \mathrm{~mm}$; and AP, $-3.00 \mathrm{~mm}, \mathrm{ML}, \pm 2.6 \mathrm{~mm}$; and DV , $-3.2 \mathrm{~mm}$. To inhibit astrocyte, CNO (Clozapine Noxide, $1 \mathrm{mg} / \mathrm{kg} /$ day, i.p., (MCE, Shanghai, China)) or saline (equal volume to $\mathrm{CNO}$, i.p.) were injected into mice according to a former study [25].

\section{Microglia depletion}

In order to evaluate the role of microglia on astrocytic activation, we eliminated microglia by feeding mice with PLX3397 according to previous study [26, 27]. Taking the patency (over 95\% depletion efficiency) into consideration, we begin to provide PLX3397 $(290 \mathrm{mg} / \mathrm{kg}$, Xietong Pharmaceutical Bio-engineering Co., JS, China) enriched chow for mice 7 days before the timepoint which microglia elimination is needed. Control mice was set by providing standard chow.

\section{LPS treatment}

To activate the microglia after etomidate sedative application, we injected LPS (i.p.) into mice according to previous study [26]. In detail, LPS (Sigma-Aldrich, MO, USA) was dissolved by saline injection at a concentration of $0.1 \mathrm{mg} / \mathrm{ml}$ and injected in mice at a dosage of $0.5 \mathrm{mg} / \mathrm{kg}$ body weight. Control mice was set by injecting with saline.

\section{Statistical Analysis}

Data were shown as mean \pm standard errors of the mean (SEM) and analyzed by GraphPad Prism software (version 8.0.1, CA, USA). Two-tailed unpaired Student's $t$ test was used for comparing the difference between two groups, and two-way repeated-measures ANOVA with Bonferroni post hoc analysis was used for the Morris water maze test. Values with $p<0.05$ were accepted as significant.

\section{Results}

Cognitive function and glial responses during the early pathological stage after etomidate application

In this study, we generated a PND mouse model by administering etomidate at a dose of $20 \mathrm{mg} / \mathrm{kg}$ body weight to 18-month-old mice and evaluated cognitive function and glial responses 1 week later (Fig. 1a). The results showed that etomidate induced significant cognitive dysfunction, including spatial and nonspatial cognitive impairments. For example, escape latency on the hidden platform test was dramatically increased (Fig 1b, c), whereas time spent in the platform quadrant (i.e., the probe test; Fig. 1d, e) and the discrimination index (i.e., novel object recognition; Fig. 1f) were significantly decreased. In addition to the behavioral data, we also observed a suppression of neuronal activity in the hippocampus (i.e., reduced frequency of the spontaneous excitatory postsynaptic current [sEPSC], spontaneous inhibitory postsynaptic current [sIPSC], miniature [m]EPSC, and miniature [m]IPSC and the amplitude of the sEPSC and sIPSC; Fig. 1g-j). Furthermore, compared to control mice, data from isolated hippocampal microglia (Fig. 1k, l; Table S2) and astrocytes (Fig 1m, n; Table S2) of model mice showed that etomidate induced an nonspecific microglial response during the early pathological stage, whereas a slight A1-like astrocyte expression profile (which is characterized by the expression of H2.T23, Serping1, H2.D1, Ggta1, Ligp1, Gbp2, Fbln5, Ugt1a, Fkbp5, Psmb8, Srgn, Amigo2 [15]) was detected in isolated hippocampal astrocytes.

\section{Cognitive function and glial responses during the late pathological stage after etomidate application}

Etomidate administration results in long-term synaptic inhibition and cognitive dysfunction in older mice [24]. Therefore, we also assessed cognitive function and glial responses 3 weeks after the injections (Fig. 2a), in 18month-old mice. We observed a notable increase in escape latency on the hidden platform test (Fig. 2b, c) and reduction in the time spent in the platform quadrant (i.e., the probe test; Fig. 2d, e) and in the discrimination index (i.e., novel object recognition; Fig. 2f). At the same time, we observed a dramatic inhibition of neuronal activity in the hippocampus (i.e., downregulated frequency of the sEPSC, sIPSC, MEPSC, and mIPSC and amplitude of the sEPSC and sIPSC; Fig. $2 \mathrm{~g}-\mathrm{j})$. Subsequently, we assessed glial responses during the late pathological stage. In contrast to the previous data from the early pathological stage, we observed a slight non-specific microglial response (Fig. 2k, 1; Table S3) and a clear A1-specific astrocyte response (Fig. 2m, n; Table S3). 
A

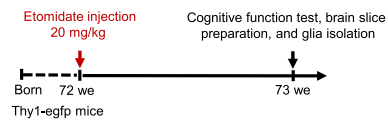

Thy1-egfp mice

B

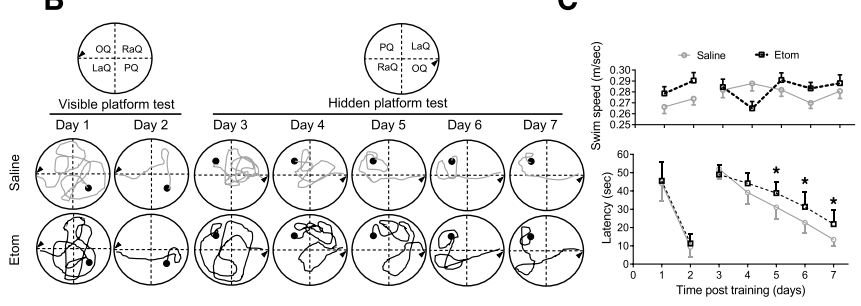

D

E

$\mathbf{F}$
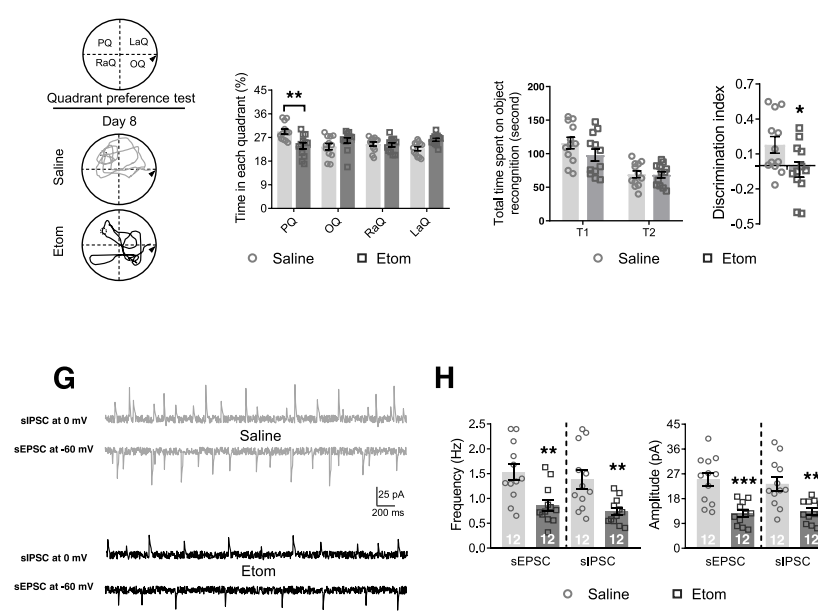

H

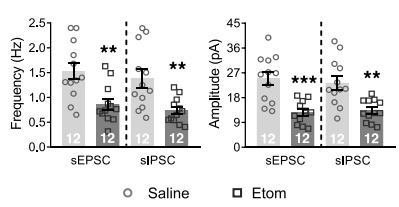

I

J
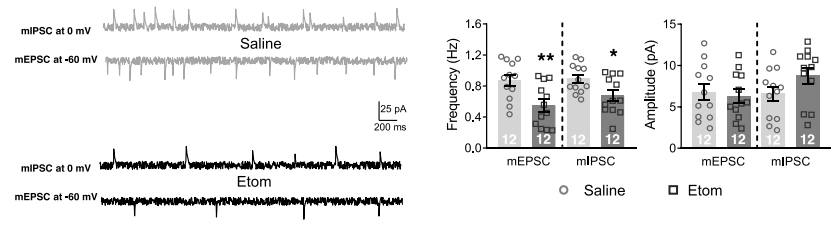

L

K

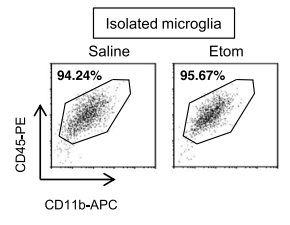

$\mathbf{L}$

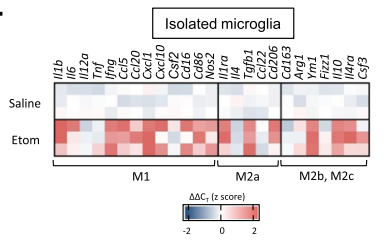



$\mathbf{N}$

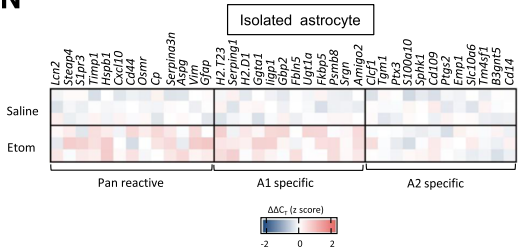

Fig. 1 (See legend on next page.) 
(See figure on previous page.)

Fig. 1 Etomidate anesthetic dosage application impairs cognition, depresses neuronal activity, and induced glial dysfunction in the early pathological stage (1 week post etomidate injection). a Graphic illustration of experimental procedure. During MWM test, we recorded swim path (b) and analyzed swim speed and latency to find platform (c) during visible and hidden platform training; (d) swim path and (e) time spent in each quadrant was investigated. $\mathbf{f}$ During NOR test, total time spent on object recognition and discrimination index were analyzed. Neuronal activities were detected by ( $\mathbf{g}$ and $\mathbf{h}$ ) sIPSC, sEPSC, (i and $\mathbf{j})$ mIPSC, and mEPSC recording and analyzing (scale bar: 200 ms, 25 pA). Selected transcripts associated with microglial polarization (microglial phenotype of M1, M2a, and M2b,2c; $\mathbf{k}$ and $\mathbf{I}$ ) and astrocyte activation (Pan-reactive, A1-specific, and A2-specific phenotype; M and N) were detected by qPCR. ( $n=12$ per group for behavioral and electrophysiological assay; $n=3$ for qPCR assay; ${ }^{*} p<0.05,{ }^{* *} p<0.01,{ }^{* * *} p<0.001$ compared with saline injected mice)

\section{Depletion of microglia during the early pathological stage improves cognitive function during the late pathological stage by suppressing A1-specific astrocyte activation}

Considering these data together led us to speculate that the A1-specific astrocyte response is a downstream reaction that requires microglial activation. Therefore, to investigate the role of microglia in astrocyte function and long-term synaptic strength, we depleted microglia by PLX3397 treatment and investigated cognitive function and glial responses 3 weeks after etomidate injections (Fig. 3a), in aged (18-month-old) mice. As expected, compared to mice fed a normal diet, mice in the PLX3397 treatment group exhibited dramatically depleted microglia and depressed inflammatory responses in the hippocampus at both the early (Fig. 3b, c; Table S4) and late pathological stages (Fig. 3d, e; Table S4). Interestingly, microglial depletion significantly improved cognitive function, reducing escape latency in the hidden platform test (Fig. 3f, g) and increasing both time spent in the platform quadrant (i.e., the probe test; Fig. 3h, i) and the discrimination index (i.e., novel object recognition; Fig. 3j). It also upregulated hippocampal neuronal activities (i.e., increased the frequency of the sEPSC, sIPSC, mEPSC, and mIPSC and the amplitude of the sEPSC and sIPSC; Fig. $3 \mathrm{k}-\mathrm{n}$ ). Moreover, data from isolated hippocampal astrocytes showed that the elimination of microglia inhibited A1-astrocyte responses (Fig. 3o, p; Table S4).

\section{Microglia depletion during the late pathological stage had no significant effect on cognitive function and astrocyte responses}

We assessed whether A1-astrocyte responses were triggered by transient microglial activation during the early pathological stage or required a constant inflammatory environment. Therefore, we depleted microglia by PLX3397 treatment during the late pathological stage and investigated cognitive function and glial responses at 3 weeks after etomidate injection (Fig. 4a), in 18-month-old mice. The data showed that more than $95 \%$ of the microglia were eliminated (Fig. 4b), and inflammatory responses were dramatically suppressed (Fig. 4c; Table S5) 3 weeks after etomidate administration. However, the cognitive function test parameters, including escape latency on the hidden platform test (Fig. 4d, e), time spent in the platform quadrant (i.e., the probe test; Fig. 4f, g), and the discrimination index (i.e., novel object recognition; Fig. 4h), as well as neuronal activity (i.e., the frequency of the sEPSC, sIPSC, mEPSC, and mIPSC and the amplitude of the sEPSC and sIPSC, mEPSC, and mIPSC; Fig. $4 \mathrm{i}-\mathrm{l}$ ) were not significantly altered. In addition, astrocyte responses were unaffected (Fig. 4m, n; Table S5).

\section{Activating microglia before etomidate sedative administration induces significant cognitive dysfunction} Next, we assessed whether activating microglia before etomidate administration triggered an A1-astrocyte response and further negative effects. We administered an intraperitoneal LPS injection to mice before the etomidate sedative injections and assessed cognitive function and astrocyte responses during the late pathological stage (Fig. 5a), in 18-month-old mice. Notably, the LPS injections promoted multiple proinflammatory transcripts in isolated microglia at the early pathological stage (Fig. 5b, c; Table S6) and concomitantly upregulated several pro-inflammatory and anti-inflammatory transcripts in isolated microglia at the late pathological stages (Fig. 5d, e; Table S6), leading to impaired cognitive function, as indicated by increased escape latency in the hidden platform test (Fig. 5f, g) and decreases in both time spent in the platform quadrant (i.e., the probe test; Fig. 5h, i) and discrimination index scores (i.e., novel object recognition; Fig. 5j). In addition, neuronal activities in the hippocampus were dramatically downregulated (i.e., reductions in the frequency of the sEPSC, SIPSC, mEPSC, and mIPSC and in the amplitude of the sEPSC and sIPSC; Fig. 5k-n). Furthermore, a slight A1-specific astrocyte response was observed (Fig. 5o, p; Table S6). 
A

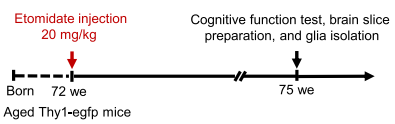

Aged Thy1-egfp mice

B

C

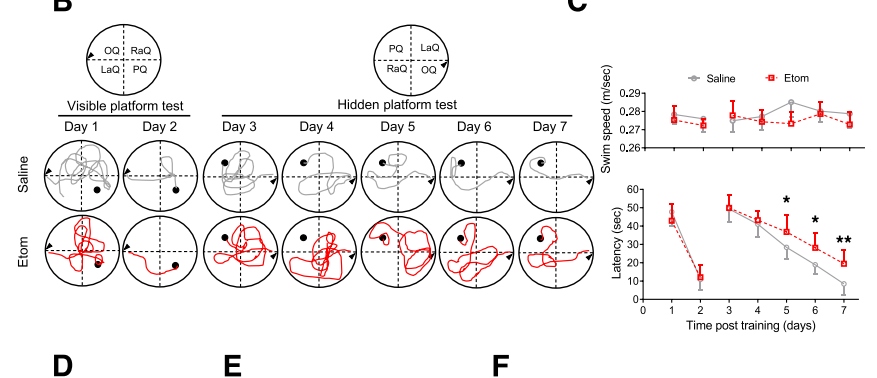

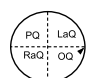
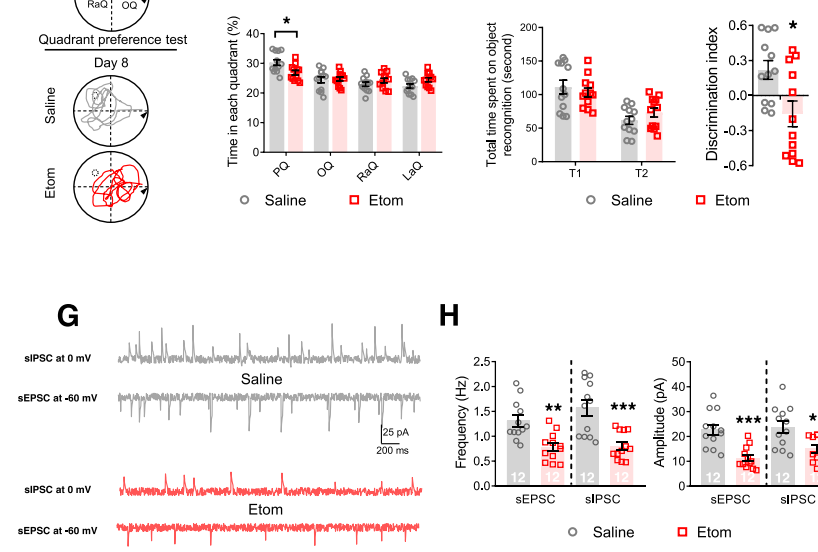

H

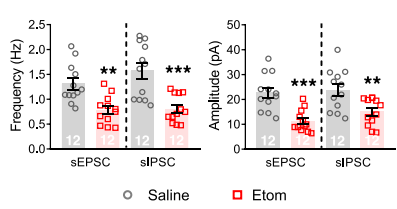

I

J

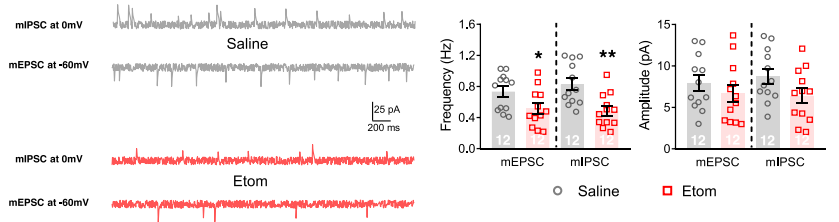

K

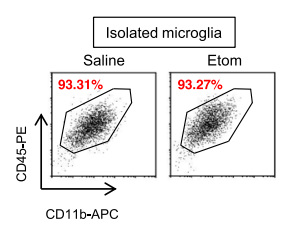

L



N
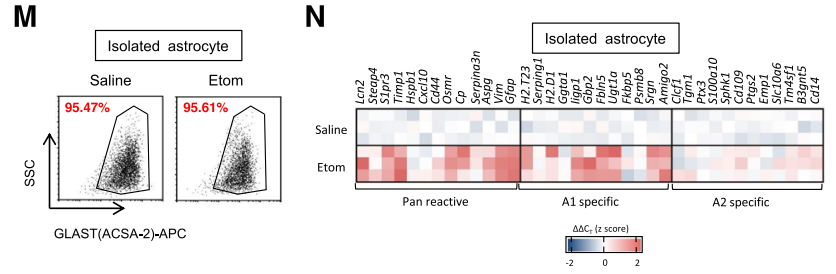

Fig. 2 (See legend on next page.) 
(See figure on previous page.)

Fig. 2 Etomidate anesthetic dosage application impairs cognition, depresses neuronal activity, and induced glial dysfunction in the late pathological stage (3 weeks post etomidate injection). a Graphic illustration of experimental procedure. During MWM test, we recorded swim path (b) and analyzed swim speed and latency to find platform (c) during visible and hidden platform training; (d) swim path and (e) time spent in each quadrant was investigated. f During NOR test, total time spent on object recognition and discrimination index were analyzed. Neuronal activities were detected by ( $\mathbf{g}$ and $\mathbf{h}$ ) sIPSC, sEPSC, (i and $\mathbf{j})$ mIPSC, and mEPSC recording and analyzing (scale bar: 200 ms, 25 pA). Selected transcripts associated with microglial polarization (microglial phenotype of M1, M2a, and M2b,2c; $\mathbf{k}$ and $\mathbf{I}$ ) and astrocyte activation (Pan-reactive, A1-specific, and A2-specific phenotype; $\mathbf{m}$ and $\mathbf{n}$ ) were detected by qPCR. ( $n=12$ per group for behavioral and electrophysiological assay; $n=3$ for qPCR assay; ${ }^{*} p<0.05,{ }^{* *} p<0.01,{ }^{* * *} p<0.001$ compared with saline injected mice)

\section{Inhibiting astrocytes reversed the microglial-activation- induced cognitive deficiencies observed after etomidate application}

Astrocyte dysfunction causes long-term synaptic inhibition and cognitive impairments [24]. However, it is unclear whether microglial-activation-induced inflammatory responses can induce these impairments without triggering A1-specific astrocyte responses. Therefore, in addition to activating microglia by LPS injection after etomidate administration, we also inhibited astrocyte activation using designer receptors exclusively activated by designer drugs (Fig. 6a), in 18-month-old mice. The results suggested that clozapine-N-oxide treatment inhibited astrocyte activation during the late pathological stage (Fig. 6b, c; Table S7) but did not change microglial responses to LPS administration in the early (Fig. 6d, e; Table S7) and late pathological stages (Fig. 6f, g; Table S7). In contrast, inhibiting astrocytes reversed microglialactivation-induced cognitive decline during the late pathological stage, reducing escape latency on the hidden platform test (Fig. 6h, i), increasing the time spent in the platform quadrant (i.e., the probe test; Fig. 6j, k), the discrimination index (i.e., novel object recognition; Fig. 6l), and synaptic inhibition (i.e., upregulating the frequency of the sEPSC, sIPSC, mEPSC, and mIPSC, as well as the amplitude of the sEPSC and sIPSC; Fig. $6 \mathrm{~m}-\mathrm{p}$ ).

\section{Discussion}

The current data suggest that both the essential and the undesired effects of anesthesia can be attributed to synaptic inhibition. Postsynaptic GABAA-R channels trigger fast and transient phasic inhibitory currents that produce amnesia, unconsciousness, and immobility, whereas extrasynaptic GABAA-R channels induce slow and persistent tonic inhibitory currents that produce undesirable effects, such as delirium and cognitive deficiencies [10]. However, many unanswered questions remain, such as (i) why does extrasynaptic GABAA-R induced longterm synaptic inhibition require astrocyte activation [14]; (ii) why are etomidate-generated GABAA-R tonic inhibitory currents alone insufficient to cause amnesia, and what other components are involved [28]; (iii) what roles do microglial and astrocyte activation play in regulating synaptic inhibition during and after anesthesia [24, 29]; (iv) is there crosstalk between the microglial and astrocyte pathways; and (v) can microglial or astrocytic inhibition alone rescue long-term synaptic inhibition and cognitive dysfunction?

\section{Non-specific microglial (early) and A1-specific astrocytic (late) responses were processed after etomidate administration in a fixed order}

Accumulating evidence has shown that glial responses promote pathogenesis and cognitive dysfunction after general anesthetics are administered. For example, cytokines (especially HMGB1, NFkB, and TNF $\alpha$ [30]) secreted from circulating or fixed mononuclear cells regulated microglial feedback reactions [31], upregulating MCP-1 expression and inflammatory cascade signaling pathways and triggering neuroinflammation in the hippocampus [32]. In addition, microglial elimination or inhibition suppressed hippocampal inflammatory responses and reduced the levels of cytokines such as IL-6, IL-1 $\beta$, and TNF $\alpha$ following anesthesia and other pathological conditions [17, 33, 34]. Moreover, the level of inositol 1,4,5-triphosphate type 2 receptor-dependent calcium was reduced in astrocytes, even after a sedative dose of anesthetic [35], and long-term inhibition of astrocytes is consistent with synaptic suppression and cognitive deficiencies [24]. Interestingly, in this study, nonspecific microglial activation occurred during the early pathological stage, together with synaptic inhibition and cognitive dysfunction, which were slightly alleviated during the late pathological stage, in an aged (18-monthold) PND mice model. In contrast to microglial activation, astrocyte expression property was not significantly altered during the early pathological stage but was stimulated in an A1-specific manner during the late pathological stage. These data suggest that (i) non-specific microglial activation may enable multiple cytokines to create an inflammatory environment, (ii) non-specific microglial responses or an inflammatory environment 


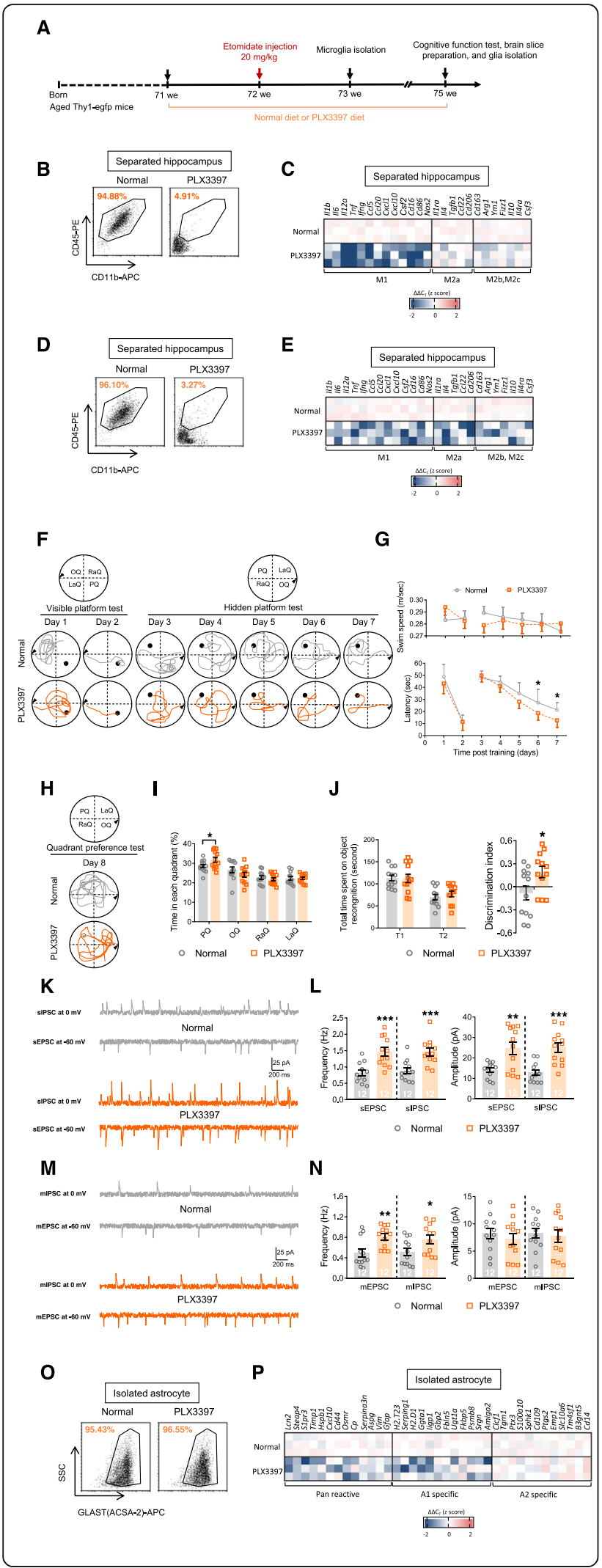

Fig. 3 Microglial depletion before etomidate injection rescues cognitive, neuronal, and glial dysfunction in the late pathological stage ( 3 weeks post etomidate injection and 4 weeks post PLX3397 diet). a Graphic illustration of experimental procedure. Flow cytometry were applied to analyze the efficiency of microglial elimination and qPCR were applied to evaluate selected transcripts associated with microglial polarization (microglial phenotype of M1, M2a, and M2b,2c) in hippocampus after PLX3397 treatment at the early stage ( 1 week post etomidate injection and 2 weeks post PLX3397 diet; $\mathbf{b}$ and $\mathbf{c}$ ) and the late stage (3 weeks post etomidate injection and 4 weeks post PLX3397 diet; $\mathbf{d}$ and e). During MWM test ( 3 weeks post etomidate injection and 4 weeks post PLX3397 diet), we recorded (f) swim path and (g) analyzed swim speed and latency to find platform during visible and hidden platform training; (h) swim path and (i) time spent in each quadrant was investigated. (j) During NOR test, total time spent on object recognition and discrimination index were analyzed. Neuronal activities were detected by (k and $\mathbf{I})$ sIPSC, sEPSC, ( $\mathbf{m}$ and $\mathbf{n}$ ) mIPSC, and mEPSC recording and analyzing (scale bar: 200 ms, 25 pA). Selected transcripts associated with astrocyte activation (Pan-reactive, A1specific, and A2-specific phenotype; $\mathbf{o}$ and $\mathbf{p}$ ) were detected by qPCR. ( $n=12$ per group for behavioral and electrophysiological assay; $n=3$ for qPCR assay; ${ }^{*} p<0.05,{ }^{* *} p<0.01,{ }^{* * *} p<0.001$ compared with normal diet mice)

may be required to trigger the A1-specific astrocytic response, and (iii) A1-specific astrocytes mediate longterm synaptic inhibition and cognitive decline.

\section{Etomidate-induced long-term synaptic inhibition is rescued after microglial depletion before the early pathological stage but not during the late pathological stage}

To evaluate the role of microglia in cognitive dysfunction after etomidate administration, we depleted microglia before anesthesia and found that this improved cognitive function and upregulated neuronal activity. This observation, together with previous results showing that inhibiting microglia activation [18] or eliminating microglia [17] dramatically enhanced cognitive function, highlights the importance of microglial activation and neuroinflammation in PND pathogenesis. However, the mechanism by which microglia mediate synaptic suppression and cognitive impairments remains unclear.

Under physiological and pathological conditions, microglia mediate synaptic suppression directly and trigger synaptic inhibition via neuroinflammation indirectly [36-38]. To determine whether microglialinduced synaptic elimination was a key factor in regulating long-term synaptic inhibition and cognitive deficiencies, we compared the cognitive function, neuronal activities, and astrocytic responses of PND mice that were treated with PLX3397 before anesthesia (depleting microglia throughout pathological progression) and PND mice (18-month-old) that were treated with 


\section{A}

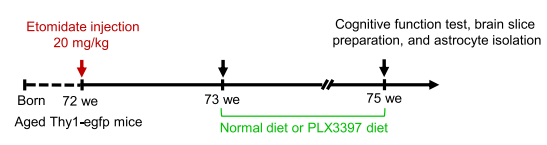

B

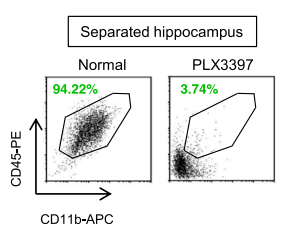

C

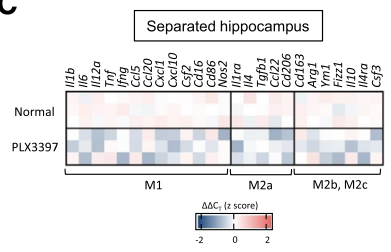

D

E

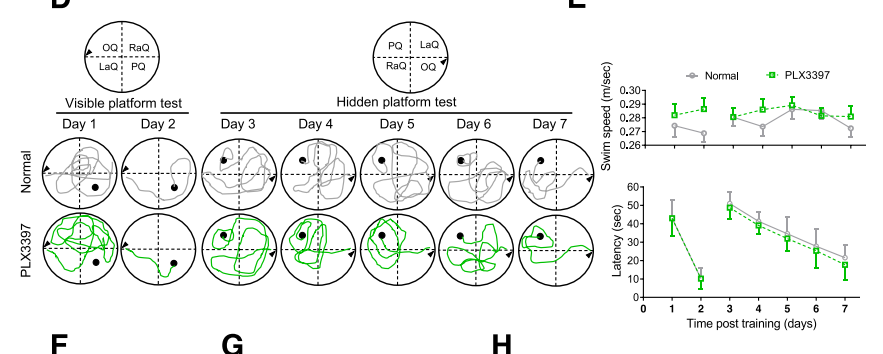

F

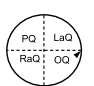

\section{H}

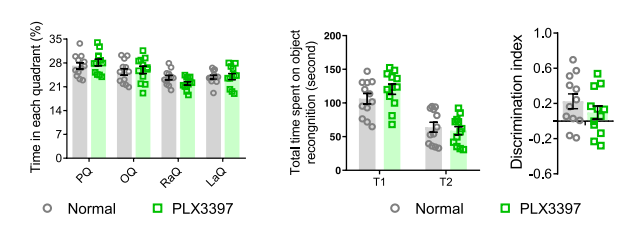

I
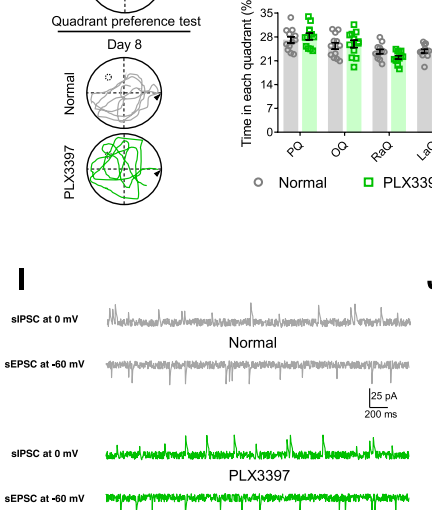

J

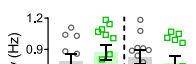

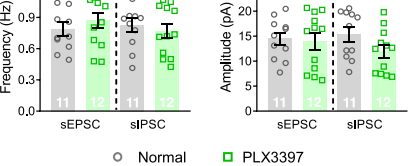

K

$\mathbf{L}$


M

N

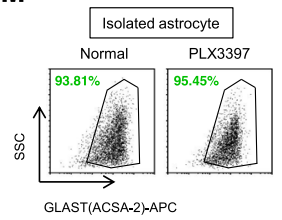

Isolated astrocyte

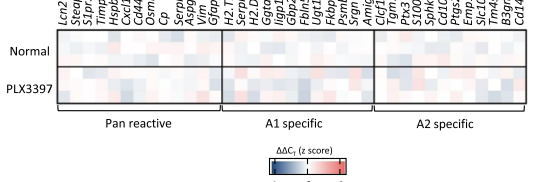

Fig. 4 (See legend on next page.) 
(See figure on previous page.)

Fig. 4 Microglial depletion at the late pathological stage of etomidate injection mice insignificantly influences cognitive, neuronal, and glial dysfunction in the late pathological stage (3 weeks post etomidate injection and 2 weeks post PLX3397 diet). a Graphic illustration of experimental procedure. $\mathbf{b}$ Flow cytometry were applied to analyze the efficiency of microglial elimination in hippocampus after PLX3397 treatment. c Selected transcripts associated with microglial polarization (microglial phenotype of M1, M2a, and M2b,2c) were detected by qPCR. During MWM test, we recorded swim path (d) and analyzed swim speed and latency to find platform (e) during visible and hidden platform training; (f) swim path and ( $\mathbf{g}$ ) time spent in each quadrant was investigated. $\mathbf{h}$ During NOR test, total time spent on object recognition and discrimination index were analyzed. Neuronal activities were detected by (i and $\mathbf{j})$ SIPSC, SEPSC, (k and $\mathbf{I})$ mIPSC, and mEPSC recording and analyzing (scale bar: 200 ms, 25 pA). Selected transcripts associated with astrocyte activation (Pan-reactive, A1-specific, and A2-specific phenotype; $\mathbf{m}$ and $\mathbf{n}$ ) were detected by qPCR. ( $n=11-12$ per group for behavioral and electrophysiological assay; $n=3$ for qPCR assay; ${ }^{*} p<0.05$, ${ }^{* *} p<0.01$, ${ }^{* * *} p<0.001$ compared with normal diet mice)

PLX3397 1 week after anesthesia (depleting microglia during the late pathological stage). In contrary to the data obtained by depleting microglia throughout pathological progression, no significant difference were observed between these groups by depleting microglia during the late pathological stage, suggesting that microglial activation may worsen the situation at the late pathological stage, but was not the only factor regulating long-term synaptic inhibition and cognitive impairment. Furthermore, astrocyte responses were not significantly affected by depleting microglia during the late pathological stage. This suggests three possibilities: (i) non-specific microglial responses during the late pathological stage of PND have no effect on long-term synaptic inhibition and cognitive dysfunction; (ii) non-specific microglial responses during the late pathological stage of PND actuate synaptic inhibition, which requires A1-specific astrocytic responses; or (iii) non-specific microglial activation during the late pathological stage of PND induces long-term synaptic inhibition and cognitive dysfunction indirectly and in an A1-specific astrocyteresponse-independent manner.

\section{Non-specific microglial activation is necessary to inhibit long-term synaptic plasticity and cognitive function in PND}

Our understanding of microglial functions depends on the application of novel cutting-edge technologies (e.g., two-photon imaging, transgenic models, and wholegenome transcriptomics). These functions include dynamic and context-dependent pro-inflammatory and anti-inflammatory gene expression, morphological changes, immune monitoring, synaptic refinement, apoptosis, and cytokine-secretion-mediated neuron survival [39]. Although microglial gene responses may not correspond to discrete cell populations, the use of the designations M1 and M2 is helpful in describing specific patterns of gene expression related to microglial activation states. Therefore, the pro-inflammatory state, designated the M1-phenotype, is characterized by the expression of IL-1 $\beta$, IL-6, IL-12p70, TNF, IFN $\gamma$, CCL5, CCL20, CXCL1, CXCL10, GM-CSF, CD16, CD86, and iNOS. The anti-inflammatory state is designated the M2phenotype and is further subdivided into the M2aphenotype (which expresses IL-1Ra, IL-4, TGF $\beta$, CCL22, and CD206) and the M2b- and M2c-phenotypes (which express CD163, Arg1, Ym1, FIZZ1, IL-10, IL-4R $\alpha$, and GCSF) [40]. We observed non-specific microglial activation after etomidate administration and tested whether etomidate-triggered non-specific activation was important for inducing long-term synaptic inhibition and cognitive dysfunction, after anesthesia in aged (18-month-old) mice.

A sedative dose of etomidate is reportedly [14] sufficient to generate phasic synaptic inhibition and short-term memory impairment after $72 \mathrm{~h}$, but fails to produce persistent long-term synaptic inhibition and cognitive impairment after 1 week. Therefore, due to this difference between the early and late stage effects of etomidate on synaptic plasticity and cognition, we investigated the role of microglial activation on inducing long-term synaptic deficiencies by administering LPS treatment before anesthesia [26, 41]. Interestingly, LPS-mediated microglial activation was sufficient to impair synaptic and cognitive function during the late pathological stage, after anesthesia in aged (18-month-old) mice. In addition, microglial depletion before etomidate administration rescued longterm synaptic inhibition, but microglial depletion during the late pathological stage failed to reverse synaptic dysfunction. These data suggest that LPS-mediated microglial activation is an essential pathological component that aggravates synaptic impairment. Furthermore, LPS-mediated microglial activation also stimulates A1-specific astrocyte responses during the late pathological stage, but whether these responses are required for long-term synaptic inhibition remains unclear.

\section{A1-specific astrocyte activation, stimulated by microglial activation, plays a critical role in long-term synaptic inhibition and cognitive deficiencies in PND}

Previous studies have emphasized the vital role of astrocyte dysfunction in causing cognitive dysfunction [24, 


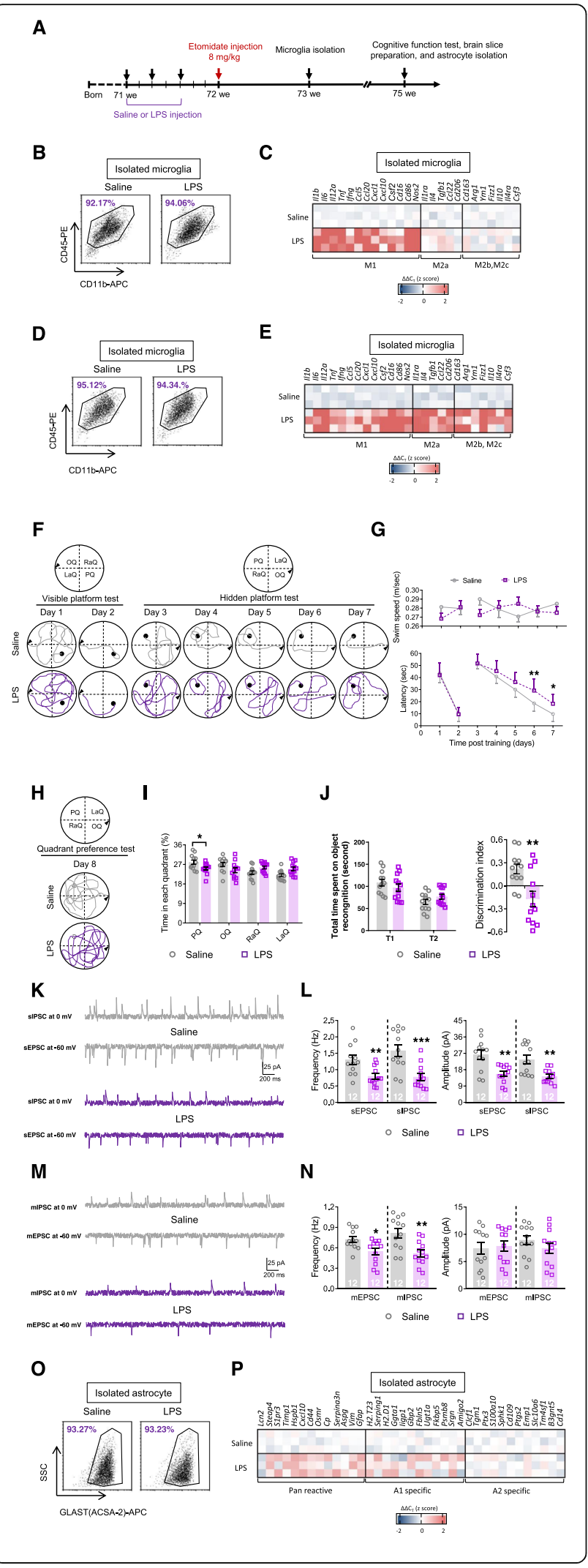

Fig. 5 LPS treatment before etomidate sedative injection impairs cognitive, neuronal, and glial function in the late pathological stage (3 weeks post etomidate sedative injection and 4 weeks post LPS injection). a Graphic illustration of experimental procedure. Flow cytometry were applied to analyze the efficiency of microglial activation and GPCR was applied to evaluate the selected transcripts associated with microglial polarization (microglial phenotype of M1, M2a, and $M 2 b, 2 c)$ in hippocampus after LPS treatment at the early stage ( 1 week post etomidate sedative injection and 2 weeks post LPS injection; b and $\mathbf{c}$ ) and late stage (3 weeks post etomidate sedative injection and 4 weeks post LPS injection; $\mathbf{d}$ and $\mathbf{e}$ ). During MWM test ( 3 weeks post etomidate sedative injection and 4 weeks post LPS injection), we recorded (f) swim path and $(\mathbf{g})$ analyzed swim speed and latency to find platform during visible and hidden platform training; (h) swim path and (i) time spent in each quadrant was investigated. $\mathbf{j}$ During NOR test, total time spent on object recognition and discrimination index were analyzed. Neuronal activities were detected by (k and $\mathbf{I})$ SIPSC, SEPSC, ( $\mathbf{m}$ and $\mathbf{n}$ ) mIPSC, and mEPSC recording and analyzing (scale bar: 200 ms, 25 pA). Selected transcripts on regulation astrocyte activation (Pan-reactive, A1-specific, and A2-specific phenotype; $\mathbf{o}$ and $\mathbf{p}$ ) were detected by GPCR. ( $n=$ 12 per group for behavioral and electrophysiological assay; $n=$ 3 for qPCR assay; ${ }^{*} p<0.05,{ }^{* *} p<0.01,{ }^{* *} p<0.001$ compared with saline injected mice)

42]. Dysfunctional astrocytes have been detected during the late pathological stage of PND. Two recent studies have highlighted the neurotoxic A1-specific astrocyte response, which is downstream of microglial activation $[15,16]$. Therefore, we investigated whether there was an association between the activation of microglia and A1-specific astrocytes in PND. Suppressing astrocyte activation during the late pathological stage prevented LPS from producing synaptic inhibition and cognitive deficiencies. This confirmed that A1-specific astrocyte responses linked early pathological microglial responses with longterm synaptic inhibition and cognitive deficiencies. However, the mechanisms of these pathways are unclear, and further studies are needed to reveal (i) the factors responsible for the initiation of proinflammatory microglial activation and neuroinflammation, (ii) whether there is a specific target for inhibiting pro-inflammatory microglial activation, (iii) how the microglia trigger A1-specific astrocytic responses, (iv) whether a particular factor can be targeted to prevent microglial activation at the early pathological stage, and (v) whether a supportive environment can be built to maintain astrocyte function. Additionally, one limitation to our study is that all experiments were carried out in male mice to eliminate the unexpected and ambiguous impact generated by gender [43, 44], thus future studies with female mice might be helpful in exploring such differences. 


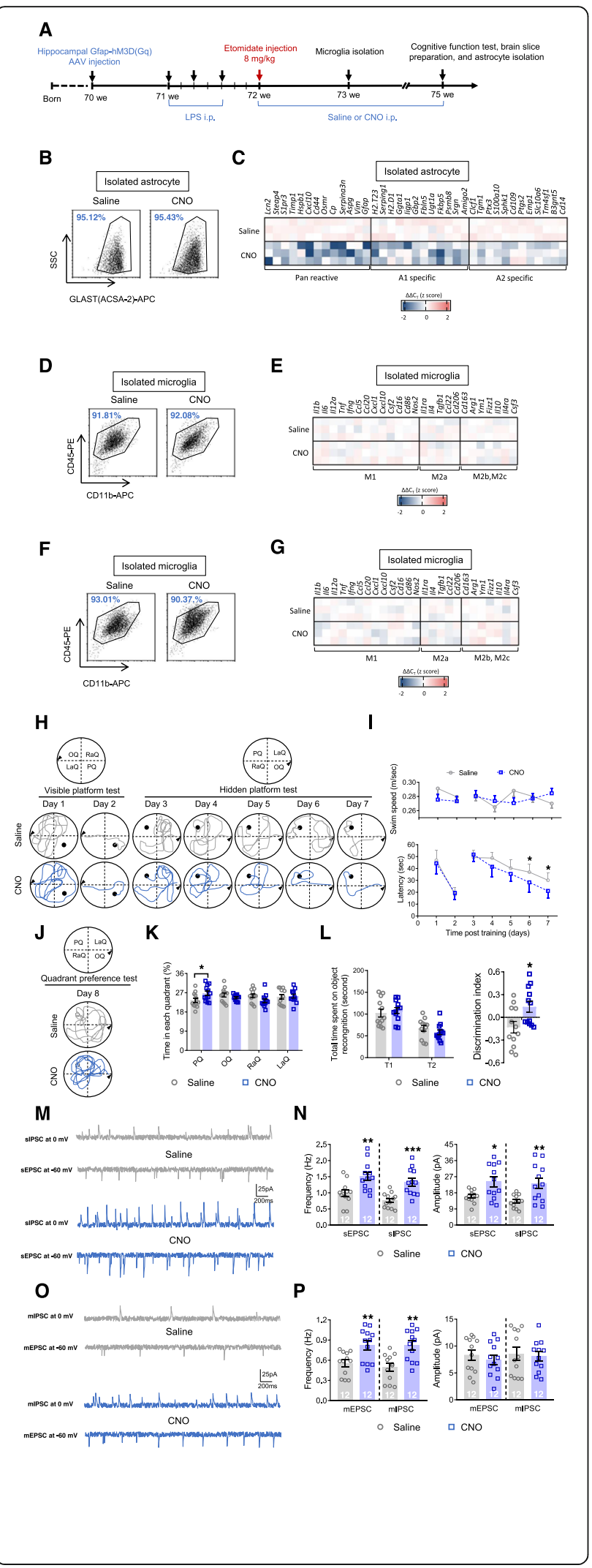

Fig. 6 Astrocytic inhibition reverses LPS treatment induced neuronal and glial dysfunction in the late pathological stage in mice after etomidate sedative injection (3 weeks post etomidate sedative injection). (a) Graphic illustration of experimental procedure. Selected transcripts on regulation astrocytic activation (Pan-reactive, A1-specific, and A2-specific phenotype; $\mathbf{b}$ and $\mathbf{c}$ ) were detected by qPCR. Flow cytometry were applied to analyze the cell purity of microglia after MACS isolation and GPCR were applied to evaluate selected transcripts associated with microglial polarization (microglial phenotype of $\mathrm{M} 1, \mathrm{M} 2 \mathrm{a}$, and $\mathrm{M} 2 \mathrm{~b}, 2 \mathrm{c}$ ) were detected by $\mathrm{qPCR}$ at the early stage ( 1 week post etomidate sedative injection; $\mathbf{d}$ and $\mathbf{e}$ ) and late stage ( 3 week post etomidate sedative injection; $\mathbf{f}$ and $\mathbf{g}$ ). During MWM test (3 weeks post etomidate sedative injection), we recorded (h) swim path and (i) analyzed swim speed and latency to find platform during visible and hidden platform training; (j) swim path and (k) time spent in each quadrant was investigated. I During NOR test, total time spent on object recognition and discrimination index were analyzed. Neuronal activities were detected by ( $\mathbf{m}$ and n) SIPSC, sEPSC, (o and $\mathbf{p}$ ) mIPSC, and mEPSC recording and analyzing (scale bar: $200 \mathrm{~ms}, 25 \mathrm{pA})$. ( $n=12$ per group for behavioral and electrophysiological assay; $n=3$ for qPCR assay; ${ }^{*} p<$ $0.05,{ }^{* *} p<0.01,{ }^{* *} p<0.001$ compared with saline injected mice)

\section{Conclusions}

In conclusion, our results show that microglial activation during the early pathological stage of PND creates an inflammatory environment and stimulates A1-specific astrocyte responses during the late pathological stage, in the aged (18-month-old) mice. This eventually induces persistent synaptic inhibition and cognitive deficiencies. These results enhance our understanding of PND pathogenesis and highlight the importance of microglia-astrocyte crosstalk during the early pathological stage.

\section{Supplementary information}

Supplementary information accompanies this paper at https://doi.org/10. 1186/s12974-020-01799-0

\section{Additional file 1. \\ Additional file 2 . \\ Additional file 3. \\ Additional file 4. \\ Additional file 5. \\ Additional file 6. \\ Additional file 7 .}

\section{Abbreviations}

aCSF: artificial cerebrospinal fluid; CCL: CCR-like protein; CXCL: Chemokine (C$X$-C motif) ligand; ED: Effective dose; GABA: $\gamma$-aminobutyric acid; GABAAR: GABA type A receptor; GM-CSF: Granulocyte-macrophage colonystimulating factor; IFN: Interferon; IL: Interleukin; iNOS: Inducible nitric oxide synthase; MACS: Magnetic-activated cell-sorting; mEPSC: miniature excitatory postsynaptic currents; mIPSC: miniature inhibitory postsynaptic currents; PND: Perioperative neurocognitive disorders; POCD: Postoperative cognitive dysfunction; sEPSC: spontaneous excitatory postsynaptic currents; sIPSC: spontaneous inhibitory postsynaptic currents; TGF: Transforming growth factor; TNF: Tumor necrosis factor

Acknowledgements

Not applicable 


\section{Authors' contributions}

$\mathrm{DL}, \mathrm{MC}$, and JF conceived and designed the study; $\mathrm{DL}$ and $\mathrm{MC}$ performed the experiments; MT analyzed the data; DL, MC, and MT wrote the draft; JF revised and edited the manuscript. The author(s) read and approved the final manuscript.

\section{Funding}

This work was supported by National Science Foundation of Shandong Province, Shandong, China (grant No. ZR2019MH133).

\section{Availability of data and materials}

The datasets used and/or analyzed details during the current study are available from the corresponding author on reasonable request.

\section{Ethics approval and consent to participate}

This study was carried out in accordance with the recommendations of the Institutional Animal Care and Use Committee (IACUC) by Shandong University, and all procedures were approved by the Administration Committee of Experimental Animals of Shandong University.

\section{Consent for publication}

Not applicable

\section{Competing interests}

The authors declare that they have no competing interests.

\section{Author details}

'Department of Anesthesiology, Qilu Hospital of Shandong University, Ji'nan 250012, China. ${ }^{2}$ Jiangsu Key Laboratory of Drug Screening, China Pharmaceutical University, Nanjing 210009, China.

\section{Received: 13 December 2019 Accepted: 31 March 2020}

Published online: 07 April 2020

\section{References}

1. Borsook D, George E, Kussman B, Becerra L. Anesthesia and perioperative stress: consequences on neural networks and postoperative behaviors. Prog Neurobiol. 2010;92:601-12.

2. Hovents IB, Schoemaker RG, van der Zee EA, Heineman E, Nyakas C, van Leeuwen BL. Thinking through postoperative cognitive dysfunction: how to bridge the gap between clinical and pre-clinical perspectives. Brain Behav Immun. 2012;26:1169-79.

3. Steinmetz J, Christensen BK, Lund T, Lohse N, Rasmussen LS. Long-term consequences of postoperative cognitive dysfunction. Anesthesiology. 2009; 110:548-55

4. Moller JT, Cluitmans P, Rasmussen LS, Houx P, Rasmussen H, Canet J, Rabbitt P, Jolles J, Larsen K, Hanning CD, Langeron O, Johnson T, Lauven PM, Kristensen PA, Biedler A, van Beem $\mathrm{H}$, Fraidakis $\mathrm{O}$, Silverstein $\mathrm{JH}$ Beneken JE, Gravenstein JS. Long-term postoperative cognitive dysfunction in the elderly ISPOCD1 study. ISPOCD investigators. International Study of Post-Operative Cognitive Dysfunction. Lancet. 1998;351(9106):857-61.

5. Barrientos RM, Frank LR, Watkins LR, Maier SF. Aging-related changes in neuroimmune-endocrine function: implications for hippocampal-dependent cognition. Horm Behav. 2012;62:219-27.

6. Hemmings HC Jr, Riegelhaupt PM, Kelz MB, Solt K, Eckenhoff RG, Orser BA Goldstein PA. Towards a comprehensive understanding of anesthetic mechanisms of action: A Decade of Discovery. Trends Pharmacol Sci. 2019; 40:464-81.

7. Maclver MB. Anesthetic agent-specific effects on synaptic inhibition. Anesth Analg. 2014;119(3):558-69.

8. Krasowski MD, Harrison NL. General anaesthetic actions on ligand-gated ion channels. Cell Mol Life Sci. 1999;55(10):1278-303.

9. Farrant $M$, Nusser Z. Variations on an inhibitory theme: phasic and tonic activation of GABA(A) receptors. Nat Rev Neurosci. 2005;6(3):215-29.

10. Engin E, Benham RS, Rudolph U. An Emerging Circuit Pharmacology of GABAA Receptors. Trends Pharmacol Sci. 2018:39(8):710-32.

11. Brickley SG, Mody I. Extrasynaptic GABA(A) receptors: their function in the CNS and implications for disease. Neuron. 2012;73(1):23-34.

12. Weir CJ, Mitchell SJ, Lambert JJ. Role of GABAA receptor subtypes in the behavioural effects of intravenous general anaesthetics. Br J Anaesth. 2017; 119(Suppl 1):i167-75
13. Belelli D, Callachan H, Hill-Venning C, Peters JA, Lambert JJ. Interaction of positive allosteric modulators with human and Drosophila recombinant GABA receptors expressed in Xenopus laevis oocytes. Br J Pharmacol. 1996; 118(3):563-76.

14. Zurek AA, Yu J, Wang DS, Haffey SC, Bridgwater EM, Penna A, Lecker I, Lei G, Chang T, Salter EW, Orser BA. Sustained increase in a5GABA $A_{A}$ receptor function impairs memory after anesthesia. J Clin Invest. 2014;124(12):543741.

15. Liddelow SA, Guttenplan KA, Clarke LE, Bennett FC, Bohlen CJ, Schirmer L, Bennett ML, Münch AE, Chung WS, Peterson TC, Wilton DK, Frouin A, Napier BA, Panicker N, Kumar M, Buckwalter MS, Rowitch DH, Dawson VL, Dawson TM, Stevens B, Barres BA. Neurotoxic reactive astrocytes are induced by activated microglia. Nature. 2017:541(7638):481-7.

16. Joshi AU, Minhas PS, Liddelow SA, Haileselassie B, Andreasson KI, Dorn GW 2nd, Mochly-Rosen D. Fragmented mitochondria released from microglia trigger $\mathrm{A} 1$ astrocytic response and propagate inflammatory neurodegeneration. Nat Neurosci. 2019;22(10):1635-48.

17. Feng $X$, Valdearcos M, Uchida Y, Lutrin D, Maze M, Koliwad SK. Microglia mediate postoperative hippocampal inflammation and cognitive decline in mice. JCl Insight. 2017;2(7):e91229.

18. Zhang D, Li N, Wang Y, Lu W, Zhang Y, Chen Y, Deng X, Yu X. Methane ameliorates post-operative cognitive dysfunction by inhibiting microglia NFKB/MAPKs pathway and promoting IL-10 expression in aged mice. Int Immunopharmacol. 2019;71:52-60.

19. Wu Y, Dou J, Wan X, Leng Y, Liu X, Chen L, Shen Q, Zhao B, Meng Q, Hou J. Histone Deacetylase Inhibitor MS-275 Alleviates Postoperative Cognitive Dysfunction in Rats by Inhibiting Hippocampal Neuroinflammation. Neuroscience. 2019:417:70-80.

20. Cheng W, Martin LJ, Elliott EM, Kim JH, Mount HT, Taverna FA, Roder JC, Macdonald JF, Bhambri A, Collinson N, Wafford KA, Orser BA. a5GABAA receptors mediate the amnestic but not sedative-hypnotic effects of the general anesthetic etomidate. J Neurosci. 2006;26(14):3713-20.

21. Feng D, Huang A, Yan W, Chen D. CD200 dysfunction in neuron contributes to synaptic deficits and cognitive impairment. Biochem Biophys Res Commun. 2019:516(4):1053-9.

22. Sheng $L$, Chen M, Cai K, Song Y, Yu D, Zhang H, Xu G. Microglial Trem2 induces synaptic impairment at early pathological stage and prevents amyloidosis at late pathological stage in APP/PS1 mice. FASEB J. 2019;33(9): 10425-42.

23. Chen MM, Qin J, Chen SJ, Yao LM, Zhang LY, Yin ZQ, Liao H. Quercetin promotes motor and sensory function recovery following sciatic nervecrush injury in C57BL/6J mice. J Nutr Biochem. 2017;46:57-67.

24. Liu Y, Liu W, Wang X, Wan Z, Gu F, Ma L, Leng Y. Hippocampal astrocyte dysfunction contributes to etomidate-induced long-lasting synaptic inhibition. Biochem Biophys Res Commun. 2019;519(4):803-11.

25. Cheng J, Umschweif G, Leung J, Sagi Y, Greengard P. HCN2 Channels in Cholinergic Interneurons of Nucleus Accumbens Shell Regulate Depressive Behaviors. Neuron. 2019;101(4):662-72.

26. Elmore MR, Najafi AR, Koike MA, Dagher NN, Spangenberg EE, Rice RA, Kitazawa M, Matusow B, Nguyen H, West BL, Green KN. Colony-stimulating factor 1 receptor signaling is necessary for microglia viability, unmasking a microglia progenitor cell in the adult brain. Neuron. 2014;82(2):380-97.

27. Rice RA, Spangenberg EE, Yamate-Morgan H, Lee RJ, Arora RP, Hernandez MX, Tenner AJ, West BL, Green KN. Elimination of Microglia Improves Functional Outcomes Following Extensive Neuronal Loss in the Hippocampus. J Neurosci. 2015;35(27):9977-89.

28. Dai S, Perouansky M, Pearce RA. Isoflurane enhances both fast and slow synaptic inhibition in the hippocampus at amnestic concentrations. Anesthesiology. 2012;116(4):816-23.

29. Baud O, Saint-Faust M. Neuroinflammation in the Developing Brain: Risk Factors, Involvement of Microglial Cells, and Implication for Early Anesthesia. Anesth Analg. 2019;128(4):718-25.

30. Terrando $\mathrm{N}$, et al. Resolving postoperative neuroinflammation and cognitive decline. Ann Neurol. 2011;70(6):986-95.

31. Hanisch UK. Microglia as a source and target of cytokines. Glia. 2002;40(2): 140-55.

32. Vacas S, Degos V, Tracey KJ, Maze M. High-mobility group box 1 protein initiates postoperative cognitive decline by engaging bone marrow-derived macrophages. Anesthesiology. 2014;120(5):1160-7.

33. Wadhwa M, Prabhakar A, Ray K, Roy K, Kumari P, Jha PK, Kishore K, Kumar S, Panjwani U. Inhibiting the microglia activation improves the spatial memory 
and adult neurogenesis in rat hippocampus during $48 \mathrm{~h}$ of sleep deprivation. J Neuroinflammation. 2017;14(1):222.

34. Fourrier C, Remus-Borel J, Greenhalgh AD, Guichardant M, Bernoud-Hubac $\mathrm{N}$, Lagarde M, Joffre C, Layé S. Docosahexaenoic acid-containing choline phospholipid modulates LPS-induced neuroinflammation in vivo and in microglia in vitro. J Neuroinflammation. 2017;14(1):170.

35. Thrane AS, Rangroo Thrane V, Zeppenfeld D, Lou N, Xu Q, Nagelhus EA, Nedergaard M. General anesthesia selectively disrupts astrocyte calcium signaling in the awake mouse cortex. Proc Natl Acad Sci U S A. 2012; 109(46):18974-9.

36. Whitelaw BS. Microglia-mediated synaptic elimination in neuronal development and disease. J Neurophysiol. 2018;119(1):1-4.

37. Schafer DP, Lehrman EK, Stevens B. The "quad-partite" synapse: microgliasynapse interactions in the developing and mature CNS. Glia. 2013;61(1):2436.

38. Hong S, Beja-Glasser VF, Nfonoyim BM, Frouin A, Li S, Ramakrishnan S, Merry KM, Shi Q, Rosenthal A, Barres BA, Lemere CA, Selkoe DJ, Stevens B.

Complement and microglia mediate early synapse loss in Alzheimer mouse models. Science. 2016;352(6286):712-6.

39. Ransohoff RM. A polarizing question: do M1 and M2 microglia exist? Nat Neurosci. 2016;19(8):987-91.

40. Lan X, Han X, Li Q, Yang QW, Wang J. Modulators of microglial activation and polarization after intracerebral haemorrhage. Nat Rev Neurol. 2017;13(7): 420-33.

41. Orihuela R, McPherson CA, Harry GJ. Microglial M1/M2 polarization and metabolic states. Br J Pharmacol. 2016;173(4):649-65.

42. Santello $\mathrm{M}$, Toni $\mathrm{N}$, Volterra A. Astrocyte function from information processing to cognition and cognitive impairment. Nat Neurosci. 2019;22(2): 154-66.

43. Schenning KJ, Murchison CF, Mattek NC, Kaye JA, Quinn JF. Sex and genetic differences in postoperative cognitive dysfunction: a longitudinal cohort analysis. Biol Sex Differ. 2019;10(1):14.

44. Kotekar N, Kuruvilla CS, Murthy V. Post-operative cognitive dysfunction in the elderly: A prospective clinical study. Indian J Anaesth. 2014;58:263-8.

\section{Publisher's Note}

Springer Nature remains neutral with regard to jurisdictional claims in published maps and institutional affiliations.

Ready to submit your research? Choose BMC and benefit from:

- fast, convenient online submission

- thorough peer review by experienced researchers in your field

- rapid publication on acceptance

- support for research data, including large and complex data types

- gold Open Access which fosters wider collaboration and increased citations

- maximum visibility for your research: over $100 \mathrm{M}$ website views per year

At $\mathrm{BMC}$, research is always in progress.

Learn more biomedcentral.com/submissions 